\title{
Effect of Stages of Maturity on Nutritive Value of Some Range Herbage Species in Low-Rainfall Woodland Savanna Southern Darfur, Sudan
}

\author{
Abu Bakr Omer Ismail1, Yousif Rizggalla Sulaiman', Faisal Awad Ahmed², \\ Hafiz Abdalla Mohamed Ali ${ }^{*}$ \\ ${ }^{1}$ Department of Animal Production, College of Natural Resources and Environmental Studies, University of \\ Juba, Khartoum, Sudan \\ ${ }^{2}$ Ministry of Science and Technology, Khartoum, Sudan \\ Email: " hafizabdo@hotmail.com
}

Received 30 August 2014; revised 21 October 2014; accepted 29 November 2014

Copyright (C) 2015 by authors and Scientific Research Publishing Inc.

This work is licensed under the Creative Commons Attribution International License (CC BY).

http://creativecommons.org/licenses/by/4.0/

(c) (i) Open Access

\begin{abstract}
Nine samples of range herbage species were collected within the grazing season using $0.5 \times 0.5 \mathrm{~m}$ quadrate as from three weeks initial pasture growth established in June across throughout up to December (two seasons), to examine the effects of age on the trend of some chemical compositions and energy changes. Analysis of variance indicated that the chemical composition of grassland from 3 weeks of age, to 12 weeks of age across to 27 weeks of age was affected by the grass age, where the metabolizable energy contents were $15.85,13.11$ and $12.36 \mathrm{MJ} / \mathrm{Kg} / \mathrm{DM}$ respectively. Hemi-celluloses and cellulose content showed a significant difference increase $(P<0.05)$ throughout the grazing season as grass age increases. The results also revealed that the Organic Matter (OM), Crude Protein (CP) and Metabolizable Energy (ME MJ/Kg DM) were significantly $(P<0.05)$ tended to be high at the beginning of growing season (mid July) $(91.05 \%, 3.8 \%$ and $8.77 \mathrm{MJ}$ respectively), then increased towards the mid of grazing season (mid September) and reduced towards the end of the grazing season (mid December).
\end{abstract}

\section{Keywords}

Chemical Composition, Grass, Grazing Season

\footnotetext{
${ }^{*}$ Corresponding author.

How to cite this paper: Ismail, A.B.O., et al. (2015) Effect of Stages of Maturity on Nutritive Value of Some Range Herbage Species in Low-Rainfall Woodland Savanna Southern Darfur, Sudan. Open Journal of Animal Sciences, 5, 1-8. 


\section{Introduction}

A major portion of grassland research and pasture development has taken place during the last 50 years to 20th century [1] [2], as such a major development occurred. Plant exploitation, improvement of pasture crops utilization quality and evaluation, which involves species and cultivars of native and naturalized grazing lands [3], this leads to development of the animal production industries worldwide.

Grasslands provide a major portion of the livestock feed for the domestic and game animals. They furnish over one-half of the total feed supply in many countries and up to 85 and 95 percent in others [4]. Grasslands cover about 24 percent of the earth land surface and occupy a great number of hectares than all crops combined [5].

Grassland in which grasses predominate still provides the basic feed requirements for growth and development of livestock particularly ruminants, their maintenance and the production of their products needs [6]. In some grazing lands, legumes and herbaceous species contribute to over all available feedstuff. Crop residues are of vast important in many regions of the tropics and subtropics [7].

A majority of these grasslands are located in regions characterized by erratic rainfall patterns and varying periods of extreme drought and often soils of poor fertility unsuited to cropping. Seasonal distribution of rainfall and soil conditions imposes a direct influence on the amount and quality of forage available during the year and indirectly affected animal performance [8]. The dry matter yields of natural grassland are considerably lower and range from 2 - 6 tones/hectare in the humid tropics and 1 - 4 tones/hectare in the arid tropics [9]. The nutritive value of herbage in tropical grazing land is inherently lower than the temperate grassland [10] and declines rapidly with advancing maturity [11]. Thus, apart from very few exceptions in the west tropics, animals in the tropical environment live for a considerable period during the year on a sub-maintenance diet.

Livestock production in the tropics can increase through increasing the output per animal and the productivity per unit area of land [12]. A major factor in increasing livestock productivity will be the improvement of animal nutrition and feed supplies, especially in the case of ruminant animals. Improved animal diseases and parasite control, breeding, and management will also be important, but initially a major emphasis must be placed on providing better nutrition [12].

More than 15 million people worldwide are dependent on pastoral nomadism razing their Herds over some 67 million $\mathrm{Km}^{2}$, which is approximately twice the cultivated area in the world [13]. It is a very important land use, most observers of the nomadic system agree that forage resources are declining as any "Equilibrium" which may have existed between nomadic herds and forage resources in the past has been destroyed. The potential for increased grassland depends upon the physical resources of any area devoted for grazing animals [14]. Obviously in areas of low rainfall, where pastures productivity is low, stocking rates will be low and output per hectare also low. Thus, a great deal of pasture research will be required to indicate the true potential of grazing lands for improvement, and continuing research is also required to insure that these potentials remain sustainable. This together with the recurring shortages of feeds interns of availability and quality, created a needs for continual researches in assessing the productivity, nutritional value of rangelands. Because live for a considerable period during the year for grazing animals depends on a sub maintenance diet [15]. However, little efforts were carried to assess the seasonal changes of the potentials of these resources. Western Sudan with its large animal population and adequately enough range resources little attempts were hardly reported to assess its seasonal potentials in terms of nutritive values [16] Therefore, the objectives of this study were to evaluate the nutritive value potentials of the grazing lands of South Darfur region by drawing a grazing calendar intervals of twenty-one days throughout the grazing season by comparing the Crude Protein (CP\%), Crude Fiber (CF\%), Nutrient Detergent Fiber (NDF\%), Acid Detergent Fiber (ADF\%), Lignin (ADL\%), and Metabolizable Energy (ME/MJ/DM).

\section{Materials and Methods}

\subsection{Site of Study}

The experiment was conducted at Ghazal Gawazat Livestock and Range Research Station, South Darfur (Latitude $11^{\circ} 28^{\prime} \mathrm{N}$; Longitude $25^{\circ} 57^{\prime} \mathrm{E}$ and altitude $485 \mathrm{~m}$ above sea level). The grazing season is divided into nine intervals (each of three weeks), as from the three weeks grass age (3 weeks), to twenty-seven (27 weeks) weeks of grass age (10th June-19th December 2004). 


\subsection{Study Design}

The total area of the station is $51.2 \mathrm{Km}^{2}$ divided by fire-lines into twenty (20) equal grazing paddocks of 2.56 $\mathrm{Km}^{2}$ each, covering an area of about 5310 ha [17]. A sample plot of one fedan $\left(4200 \mathrm{~m}^{2}\right)$, in paddock No. 20 was selected as the study site. The criteria for its selection included its ecological representation of all soil types and accessibility. The site was fenced and was guarded against trespassing and animal grazing throughout the study period. The study commenced when pasture showed initial growth and continued for 27 weeks with samples being harvested every 3 weeks, as from first rains in 18th June to first July when grass sward was established, then followed by an interval of 21 days according to the methods described by [18] to facilitate for analyzing the nutritive value of grassland.

The range samples were collected from within the study site by manual clipping of enough amounts of grasses using the $0.5 \times 0.5 \mathrm{~m}$ quadrate with scissors. The samples were clipped $5 \mathrm{cms}$ above the ground level according to [16] regrowth areas previously clipped was not sampled again. The samples were subjected to sun dry, and composite samples were kept for subsequent analysis. The chemical analysis was performed according to [19]-[21] method for Neutral Detergent Fiber analysis.

\subsection{Statistical Analysis}

The effect of advance range age on nutritive value in terms of dry matter (DM\%), crude protein (CP\%), crude fiber (CF\%), nutrient detergent fiber(NDF\%), acid detergent fiber (ADF\%), cellulose and lignin decrease or increase was investigated. Also the trend in metabolizable energy content was calculated after [22]. The data were subjected to least square analysis of Variance [23] and the means were tested for significance by multiple range test [24] as a pes hoc (means separation).

\section{Results}

As shown in Table 1 the advance in grass age through the season affected feeding value grades of range herbage. The organic matter, crude protein, crude fiber, neutral detergent fiber, acid detergent fiber, lignin and metabolizable energy was significantly $(\mathrm{P}<0.05)$ varies across the grazing season ,the organic matter tends to be higher in the first three weeks after the sward was established, than in the last twenty seven weeks of age. Also the

Table 1. Variation in nutritive value of the range herbage throughout the 1st grazing season from 3rd weeks to 27th weeks age of grassland as grazing calendar in the low rainfall woodland savanna.

\begin{tabular}{|c|c|c|c|c|c|c|c|c|c|c|c|c|}
\hline \multicolumn{13}{|c|}{ Grass Age in Weeks } \\
\hline Chemical Analysis & 3 & 6 & 9 & 12 & 15 & 18 & 21 & 24 & 27 & $\begin{array}{c}\text { Overall } \\
\text { Mean }\end{array}$ & SEM & LSD \\
\hline DM\% & $80.58^{d}$ & $82.04^{\mathrm{d}}$ & $84.00^{c}$ & $90.23^{b}$ & $92.30^{\mathrm{b}}$ & $96.19^{\mathrm{a}}$ & $91.47^{\mathrm{b}}$ & $91.10^{\mathrm{b}}$ & $91.87^{\mathrm{b}}$ & 88.65 & 1.01 & $* *$ \\
\hline Ash\% & $8.95^{\mathrm{e}}$ & $10.59^{d}$ & $9.98^{\mathrm{e}}$ & $8.88^{\mathrm{e}}$ & $8.60^{\mathrm{e}}$ & $13.00^{\mathrm{b}}$ & $12.19^{\mathrm{c}}$ & $14.18^{\mathrm{a}}$ & $12.07^{\mathrm{c}}$ & 10.94 & 0.52 & $* *$ \\
\hline ОМ\% & $91.05^{\mathrm{a}}$ & $89.41^{\mathrm{a}}$ & $89.98^{\mathrm{a}}$ & $91.13^{\mathrm{a}}$ & $91.40^{\mathrm{a}}$ & $87.50^{c}$ & $87.81^{\mathrm{c}}$ & $85.83^{e}$ & $87.93^{\mathrm{b}}$ & 89.11 & 0.59 & $*$ \\
\hline $\mathrm{CP} \%$ & $3.89^{d}$ & $4.06^{\mathrm{d}}$ & $7.90^{\mathrm{a}}$ & $8.80^{\mathrm{a}}$ & $8.60^{\mathrm{a}}$ & $6.91^{\mathrm{b}}$ & $5.21^{c}$ & $5.08^{\mathrm{c}}$ & $4.08^{\mathrm{d}}$ & 6.06 & 0.27 & $* *$ \\
\hline $\mathrm{CF} \%$ & $21.25^{f}$ & $19.38^{f}$ & $22.53^{f}$ & $34.47^{c}$ & $34.71^{\mathrm{c}}$ & $38.79^{\mathrm{a}}$ & $36.05^{\mathrm{b}}$ & $32.86^{\mathrm{d}}$ & $28.96^{c}$ & 29.85 & 0.71 & $* * *$ \\
\hline $\mathrm{EE} \%$ & $6.63^{c}$ & $5.05^{d}$ & $5.95^{d}$ & $7.86^{\mathrm{c}}$ & $4.28^{\mathrm{e}}$ & $3.96^{\mathrm{e}}$ & $5.17^{d}$ & $10.18^{\mathrm{b}}$ & $12.90^{\mathrm{a}}$ & 6.88 & 0.41 & $* * *$ \\
\hline NFE\% & $45.13^{\mathrm{a}}$ & $46.26^{\mathrm{a}}$ & $48.94^{b}$ & $40.78^{c}$ & $34.26^{\mathrm{e}}$ & $34.88^{c}$ & $37.82^{\mathrm{d}}$ & $30.66^{\mathrm{e}}$ & $42.55^{\mathrm{b}}$ & 38.81 & 1.29 & $* * *$ \\
\hline NDF\% & $52.30^{\mathrm{d}}$ & $59.95^{\mathrm{c}}$ & $61.79^{c}$ & $66.59^{c}$ & $69.60^{\mathrm{b}}$ & $73.53^{\mathrm{a}}$ & $53.82^{\mathrm{d}}$ & $53.60^{d}$ & $56.65^{d}$ & 60.09 & 0.93 & $* * *$ \\
\hline Hemi-Cellulose\% & $8.62^{\mathrm{e}}$ & $27.32^{\mathrm{b}}$ & $26.17^{\mathrm{b}}$ & $34.44^{\mathrm{a}}$ & $28.60^{b}$ & $22.88^{c}$ & $19.69^{d}$ & $15.29^{d}$ & $9.25^{\mathrm{e}}$ & 21.31 & 2.42 & $* * *$ \\
\hline $\mathrm{ADF} \%$ & $36.65^{d}$ & $37.64^{\mathrm{d}}$ & $35.13^{d}$ & $32.15^{\mathrm{e}}$ & $41.05^{\mathrm{c}}$ & $51.16^{\mathrm{a}}$ & $34.13^{\mathrm{d}}$ & $38.24^{\mathrm{d}}$ & $47.40^{\mathrm{b}}$ & 39.28 & 0.94 & $* * *$ \\
\hline Cellulose\% & $26.28^{c}$ & $24.00^{c}$ & $25.88^{\mathrm{C}}$ & $24.00^{c}$ & $36.71^{b}$ & $86.04^{\mathrm{a}}$ & $16.97^{\mathrm{d}}$ & $16.18^{d}$ & $12.55^{\mathrm{e}}$ & 23.73 & 1.48 & $* * *$ \\
\hline Lignin\% & $7.81^{\mathrm{e}}$ & $8.31^{\mathrm{e}}$ & $8.26^{\mathrm{e}}$ & $8.15^{e}$ & $9.34^{\mathrm{e}}$ & $15.12^{\mathrm{d}}$ & $17.16^{\mathrm{c}}$ & $22.06^{\mathrm{b}}$ & $34.85^{\mathrm{a}}$ & 15.45 & 0.52 & $* * *$ \\
\hline ME MJ/kg/DM & $15.85^{\mathrm{a}}$ & $12.87^{\mathrm{c}}$ & $12.25^{\mathrm{c}}$ & $13.11^{\mathrm{b}}$ & $12.02^{\mathrm{C}}$ & $9.57^{\mathrm{e}}$ & $11.06^{\mathrm{d}}$ & $12.99^{c}$ & $12.36^{\mathrm{c}}$ & 12.45 & 0.56 & $* *$ \\
\hline
\end{tabular}

a, b, c, d, and e values on the same row with different superscripts differ significantly. SE = Standard Error. LSD = Least Significant Difference. ${ }^{*}=$ Significant at $(\mathrm{P}<0.05) .{ }^{* *}=$ Significant at $(\mathrm{P}<0.01) .{ }^{* * *}=$ Significant at $(\mathrm{P}<0.001) . \mathrm{DM}=$ Dry Matter, OM $=$ Organic Matter, $\mathrm{CP}=\mathrm{Crude}$ Protein, $\mathrm{CF}=\mathrm{Crude}$ Fiber, $\mathrm{EE}=$ Ether Extract, NFE = Nitrogen Free Extract, NDF $=$ Neutral Detergent Fiber, ADF = Acid Detergent Fiber, ME $=$ Metabolizable Energy . 
crude protein significantly $(\mathrm{P}<0.05)$ was higher during the same time and tends to declines towards the end of the grazing season. On the other hand, crude fiber, neutral detergent fiber, and acid detergent fiber significantly ( $P<0.001$ ) higher towards the eighteen weeks of age than in the first three weeks of age. The metabolizable energy was significantly $(\mathrm{P}<0.05)$ higher at the early grass age ( 3 weeks, $15.85 \mathrm{MJ} / \mathrm{Kg}$ ) than in late grass age 27 weeks of age $(12.36 \mathrm{MJ} / \mathrm{Kg})$.

Table 2 shows that on monthly basis intervals during the season 2004, where the dry matter, crude fiber and neutral detergent fiber was significantly $(\mathrm{P}<0.01)$ higher in mid October than in mid December, while crude protein level in mid September is better than both in the first July and mid December. On the other hand Lignin tends to be high in mid December (34.85\%) than mid August and mid October (8.26\% and 15.82\% respectively).

As shown also in Table 3 and Table 4 for the second grazing season 2005, the organic matter, crude protein, neutral detergent fiber and lignin $(\%)$ was significantly $(\mathrm{P}<0.05)$ varies throughout the season following the trend of variability both in weeks and monthly basis as in the first season as shown in Table 1 and Table 2 respectively. Metabolizable energy ( $\mathrm{MJ} / \mathrm{Kg}$ ) was also significantly high in the first early grazing season in the 15th weeks of age and tends to decline towards the 27th weeks of age. Also in monthly basis it tends to be high in mid September and declines towards the mid of December (8.81, 6.16 MJ/Kg respectively).

Table 5 reveals the comparisons of the range grasses nutritive value for the two seasons. Organic matter, neutral detergent fiber and cellulose were significantly $(\mathrm{P}<0.05)$ higher in 1st grazing season than in 2nd grazing season. On the other hands, acid detergent fiber and lignin tend to be significantly $(\mathrm{P}<0.05)$ higher in 2nd grazing season than in 1st grazing season. Metabolizable energy $(\mathrm{MJ} / \mathrm{Kg}$ ), crude protein $(\mathrm{CP})$ and crude fiber (CF) showed no significant differences between the two groups.

\section{Discussion}

The chemical composition of range grassland is influenced by different factors such as grass age, soil type and remarkably the precipitation intensity or season [16]. The range grass consist of several types of species of different nature being herbs or grasses or phorbs combined together in a well established sward to meet the basic animal requirements in terms of their available nutrients contents during the grazing season.

Table 2. Monthly variation in nutritive value of the range herbage during the 1st grazing season.

\begin{tabular}{|c|c|c|c|c|c|c|c|c|c|}
\hline \multicolumn{10}{|c|}{ Grass Age Intervals (Month) } \\
\hline Nutrients & $\begin{array}{l}\text { First } \\
\text { July }\end{array}$ & $\begin{array}{c}\text { Mid } \\
\text { August }\end{array}$ & $\begin{array}{c}\text { Mid } \\
\text { September }\end{array}$ & $\begin{array}{c}\text { Mid } \\
\text { October }\end{array}$ & $\begin{array}{c}\text { Mid } \\
\text { November }\end{array}$ & $\begin{array}{c}\text { Mid } \\
\text { December }\end{array}$ & $\begin{array}{c}\text { Overall } \\
\text { Mean }\end{array}$ & SEM & LSD \\
\hline DM\% & $80.58^{\mathrm{d}}$ & $84.00^{\mathrm{d}}$ & $90.23^{\mathrm{c}}$ & $96.19^{\mathrm{a}}$ & $91.47^{\mathrm{b}}$ & $91.87^{\mathrm{b}}$ & 88.65 & 1.01 & ** \\
\hline Ash\% & $8.95^{\mathrm{d}}$ & $9.98^{\mathrm{c}}$ & $8.88^{\mathrm{d}}$ & $13.00^{\mathrm{a}}$ & $12.19^{b}$ & $12.07^{\mathrm{b}}$ & 10.94 & 0.52 & * \\
\hline OM\% & $91.05^{\mathrm{a}}$ & $89.98^{\mathrm{b}}$ & $91.13^{\mathrm{a}}$ & $87.50^{c}$ & $87.81^{\mathrm{c}}$ & $87.93^{c}$ & 89.11 & 0.59 & ** \\
\hline $\mathrm{CP} \%$ & $3.82^{\mathrm{e}}$ & $7.90^{\mathrm{e}}$ & $8.80^{\mathrm{a}}$ & $6.91^{\mathrm{c}}$ & $5.21^{\mathrm{d}}$ & $4.03^{\mathrm{e}}$ & 6.06 & 0.27 & ** \\
\hline $\mathrm{CF} \%$ & $21.25^{\mathrm{e}}$ & $22.53^{\mathrm{e}}$ & $34.17^{\mathrm{c}}$ & $38.79^{\mathrm{a}}$ & $36.05^{\mathrm{b}}$ & $28.96^{\mathrm{d}}$ & 29.85 & 0.71 & * \\
\hline $\mathrm{EE} \%$ & $6.63^{\mathrm{a}}$ & $5.95^{\mathrm{e}}$ & $7.86^{\mathrm{a}}$ & $3.96^{\mathrm{d}}$ & $5.17^{c}$ & 12.90 & 6.88 & 0.41 & * \\
\hline NFEE\% & $45.13^{e}$ & $43.94^{\mathrm{b}}$ & $40.78^{\mathrm{c}}$ & $34.88^{\mathrm{e}}$ & $37.82^{\mathrm{d}}$ & $42.55^{\mathrm{b}}$ & 38.81 & 1.29 & * \\
\hline NDF\% & $45.34^{e}$ & $61.79^{c}$ & $66.59^{\mathrm{b}}$ & $73.53^{\mathrm{a}}$ & $58.84^{\mathrm{d}}$ & $56.65^{\mathrm{d}}$ & 60.09 & 0.39 & ** \\
\hline Hemi-Cellulose\% & $8.62^{\mathrm{c}}$ & $26.17^{\mathrm{c}}$ & $34.44^{\mathrm{a}}$ & $22.32^{\mathrm{b}}$ & $19.69^{d}$ & $9.25^{\mathrm{e}}$ & 21.31 & 2.42 & ** \\
\hline ADF\% & $36.65^{\mathrm{c}}$ & $35.13^{c}$ & $32.15^{\mathrm{d}}$ & $51.16^{\mathrm{a}}$ & $34.13^{\mathrm{d}}$ & $47.46^{\mathrm{b}}$ & 39.28 & 0.94 & * \\
\hline Cellulose\% & $26.28^{\mathrm{b}}$ & $25.88^{b}$ & $24.20^{c}$ & $36.04^{\mathrm{a}}$ & $16.97^{\mathrm{d}}$ & $12.55^{\mathrm{e}}$ & 23.73 & 1.48 & $*$ \\
\hline Lignin\% & $7.81^{\mathrm{e}}$ & $8.26^{\mathrm{d}}$ & $8.15^{\mathrm{d}}$ & $15.82^{c}$ & $17.16^{\mathrm{b}}$ & $34.85^{\mathrm{a}}$ & 15.45 & 0.52 & ** \\
\hline ME MJ/kg/DM & $8.85^{\mathrm{a}}$ & $8.11^{\mathrm{ab}}$ & $8.62^{\mathrm{b}}$ & $6.76^{\mathrm{c}}$ & $6.47^{\mathrm{d}}$ & $6.40^{\mathrm{d}}$ & 12.45 & 0.56 & ** \\
\hline
\end{tabular}

a, b, c, d, and e values on the same row with different superscripts differ significantly. SE $=$ Standard Error. LSD $=$ Least Significant Difference. ${ }^{*}=$ Significant at $(\mathrm{P}<0.05){ }^{* *}=$ Significant $(\mathrm{P}<0.01) . \mathrm{DM}=$ Dry Matter, $\mathrm{OM}=$ Organic Matter, $\mathrm{CP}=$ Crude Protein, $\mathrm{CF}=\mathrm{Crude}$ Fiber, EE $=$ Ether Extract, NFE = Nitrogen Free Extract, NDF $=$ Neutral Detergent Fiber, ADF $=$ Acid Detergent Fiber, ME $=$ Metabolizable Energy. 
Table 3. Variation in the nutritive value of the range herbage throughout the 2nd grazing season from 3 weeks age to 27th weeks of age of grassland as grazing calendar in the low rainfall woodland savanna.

\begin{tabular}{|c|c|c|c|c|c|c|c|c|c|c|c|c|}
\hline \multicolumn{13}{|c|}{ Grass Age in Weeks } \\
\hline $\begin{array}{l}\text { Chemical } \\
\text { Analysis }\end{array}$ & 3 & 6 & 9 & 12 & 15 & 18 & 21 & 24 & 27 & $\begin{array}{c}\text { Overall } \\
\text { Mean }\end{array}$ & SEM & LSD \\
\hline DM\% & $80.88^{c}$ & $82.75^{c}$ & $91.49^{\mathrm{cc}}$ & $90.77^{\mathrm{d}}$ & $9525^{\mathrm{b}}$ & $95.09^{\mathrm{b}}$ & $91.17^{\mathrm{d}}$ & $93.93^{c}$ & $96.60^{\mathrm{a}}$ & 89.77 & 1.22 & ** \\
\hline Ash\% & 13.15 & 11.91 & 11.06 & 11.63 & 9.17 & 12.23 & 10.72 & 12.19 & 13.03 & 11.73 & 0.99 & NS \\
\hline OM\% & 86.85 & 88.09 & 88.64 & 87.38 & 89.29 & 87.78 & 89.29 & 87.81 & 86.67 & 88.42 & 0.68 & NS \\
\hline СР\% & $5.05^{\mathrm{d}}$ & $5.05^{\mathrm{d}}$ & $7.72^{\mathrm{c}}$ & $8.09^{\mathrm{b}}$ & $9.58^{\mathrm{a}}$ & $7.28^{\mathrm{c}}$ & $5.08^{\mathrm{d}}$ & $3.75^{\mathrm{d}}$ & $4.49^{\mathrm{d}}$ & 6.21 & 0.48 & * \\
\hline $\mathrm{CF} \%$ & $22.26^{\mathrm{d}}$ & $24.00^{\mathrm{d}}$ & $24.23^{\mathrm{d}}$ & $31.26^{c}$ & $44.03^{\mathrm{a}}$ & $24.25^{\mathrm{d}}$ & $41.46^{\mathrm{b}}$ & $33.73^{c}$ & $23.70^{\mathrm{d}}$ & 29.88 & 1.14 & $* * *$ \\
\hline $\mathrm{EE} \%$ & $5.30^{c}$ & $6 . .03^{\mathrm{b}}$ & $7.20^{\mathrm{a}}$ & $4.38^{\mathrm{d}}$ & $5.88^{\mathrm{c}}$ & $3.79^{\mathrm{d}}$ & $6.66^{\mathrm{c}}$ & $3.61^{\mathrm{d}}$ & $2.80^{\mathrm{c}}$ & 4.96 & 0.38 & $*$ \\
\hline NFEE\% & $37.26^{\mathrm{c}}$ & $34.18^{\mathrm{d}}$ & $34.35^{\mathrm{d}}$ & $37.70^{c}$ & $48.25^{\mathrm{a}}$ & $38.26^{\mathrm{b}}$ & $38.80^{\mathrm{b}}$ & $37.80^{c}$ & $49.83^{\mathrm{a}}$ & 39.60 & 1.07 & ** \\
\hline NDF\% & $36.23^{c}$ & $59.80^{\mathrm{b}}$ & $54.68^{\mathrm{c}}$ & $45.38^{d}$ & $47.50^{\mathrm{d}}$ & $64.79^{\mathrm{a}}$ & $61.25^{\mathrm{b}}$ & $63.38^{\mathrm{a}}$ & $64.05^{\mathrm{a}}$ & 54.67 & 1.02 & $* * *$ \\
\hline Hemi-Cellulose\% & $4.48^{\mathrm{f}}$ & $21.09^{\mathrm{b}}$ & $8.27^{\mathrm{f}}$ & $6.99^{\mathrm{f}}$ & $8.99^{f}$ & $19.12^{c}$ & $11.90^{\mathrm{c}}$ & $15.22^{\mathrm{d}}$ & $30.11^{\mathrm{a}}$ & 14.00 & 1.42 & $* * *$ \\
\hline $\mathrm{ADF} \%$ & $31.75^{\mathrm{d}}$ & $38.72^{\mathrm{b}}$ & $46.36^{\mathrm{a}}$ & $38.39^{\mathrm{b}}$ & $35.81^{\mathrm{c}}$ & $46.67^{\mathrm{a}}$ & $49.35^{\mathrm{a}}$ & $45.66^{\mathrm{a}}$ & $33.94^{c}$ & 40.74 & 1.48 & $* *$ \\
\hline Cellulose\% & $14.05^{\mathrm{d}}$ & $12.98^{\mathrm{d}}$ & $29.55^{\mathrm{a}}$ & $23.37^{\mathrm{b}}$ & $14.50^{\mathrm{d}}$ & $30.44^{\mathrm{a}}$ & $24.21^{\mathrm{b}}$ & $24.99^{\mathrm{b}}$ & $20.09^{c}$ & 21.47 & 2.32 & $* *$ \\
\hline Lignin\% & $9.70^{\mathrm{e}}$ & $12.49^{\mathrm{c}}$ & $16.81^{\mathrm{d}}$ & $15.02^{\mathrm{d}}$ & $21.34^{c}$ & $22.24^{\mathrm{c}}$ & $25.14^{\mathrm{b}}$ & $31.56^{\mathrm{a}}$ & $33.86^{\mathrm{a}}$ & 19.24 & 1.44 & ** \\
\hline ME MJ/kg/DM & $12.85^{\mathrm{b}}$ & $12.06^{\mathrm{b}}$ & $12.40^{\mathrm{b}}$ & $11.37^{c}$ & $13.66^{\mathrm{a}}$ & $9.39^{d}$ & $11.86^{\mathrm{c}}$ & $9.65^{d}$ & $9.80^{\mathrm{d}}$ & 11.44 & 0.41 & * \\
\hline
\end{tabular}

a, b, c, d, e, and f values on the same row with different superscripts differ significantly. SED = Standard Error. LSD = Least Significant Difference. NS = Not Significant, ${ }^{*}=$ Significant at $(\mathrm{P}<0.05) .{ }^{* *}=$ Significant at $(\mathrm{P}<0.01) .{ }^{* * *}=$ Significant at $(\mathrm{P}<0.001) . \mathrm{DM}=\mathrm{Dry}$ Matter, OM $=$ Organic Matter, $\mathrm{CP}=\mathrm{Crude}$ Protein, $\mathrm{CF}=$ Crude Fiber, EE $=$ Ether Extract, NFE = Nitrogen Free Extract, NDF = Neutral Detergent Fiber, ADF $=$ Acid Detergent Fiber, ME $=$ Metabolizable Energy.

Table 4. Monthly variation in nutritive value of the range herbage during the 2nd grazing season.

\begin{tabular}{|c|c|c|c|c|c|c|c|c|c|}
\hline \multicolumn{10}{|c|}{ Grass Age Intervals } \\
\hline Chemical Composition & $\begin{array}{l}\text { First } \\
\text { July }\end{array}$ & $\begin{array}{c}\text { Mid } \\
\text { August }\end{array}$ & $\begin{array}{c}\text { Mid } \\
\text { September }\end{array}$ & $\begin{array}{c}\text { Mid } \\
\text { October }\end{array}$ & $\begin{array}{c}\text { Mid } \\
\text { November }\end{array}$ & $\begin{array}{c}\text { Mid } \\
\text { December }\end{array}$ & $\begin{array}{l}\text { Overall } \\
\text { Mean }\end{array}$ & SEM & LSD \\
\hline DM\% & $80.88^{d}$ & $89.49^{c}$ & $90.77^{\mathrm{b}}$ & $95.09^{\mathrm{a}}$ & $91.17^{\mathrm{b}}$ & $96.60^{\mathrm{a}}$ & $89.77^{\mathrm{c}}$ & 1.22 & $*$ \\
\hline Ash\% & 13.45 & 11.06 & 11.63 & 12.23 & 10.72 & 13.03 & 11.73 & 0.99 & NS \\
\hline $\mathrm{CP} \%$ & $5.05^{\mathrm{c}}$ & $7.72^{\mathrm{b}}$ & $8.09^{\mathrm{a}}$ & $7.28^{\mathrm{b}}$ & $5.08^{\mathrm{c}}$ & $4.40^{\mathrm{d}}$ & 6.21 & 0.48 & $* *$ \\
\hline $\mathrm{CF} \%$ & $22.26^{\mathrm{d}}$ & $24.22^{\mathrm{C}}$ & $31.26^{\mathrm{b}}$ & $24.25^{c}$ & $41.46^{\mathrm{a}}$ & $23.70^{\mathrm{d}}$ & 29.88 & 1.14 & $* *$ \\
\hline $\mathrm{EE} \%$ & $5.30^{\mathrm{b}}$ & $7.20^{\mathrm{a}}$ & $4.33^{c}$ & $3.39^{d}$ & $5.66^{b}$ & $2.80^{\mathrm{e}}$ & 4.96 & 0.38 & $* *$ \\
\hline NFEE\% & $37.26^{\mathrm{c}}$ & $34.35^{d}$ & $37.70^{\mathrm{c}}$ & $38.26^{\mathrm{b}}$ & $38.80^{\mathrm{b}}$ & $49.33^{\mathrm{a}}$ & 39.60 & 1.07 & $* *$ \\
\hline Hemi-Cellulose\% & $4.48^{\mathrm{f}}$ & $8.27^{\mathrm{d}}$ & $6.99^{\mathrm{e}}$ & $19.12^{\mathrm{b}}$ & $11.90^{\mathrm{c}}$ & $30.11^{\mathrm{a}}$ & 14.00 & 1.42 & * \\
\hline$(\mathrm{ADF} \%)$ & $31.75^{\mathrm{e}}$ & $46.36^{\mathrm{b}}$ & $38.39^{c}$ & $46.67^{\mathrm{b}}$ & $49.35^{\mathrm{a}}$ & $33.94^{d}$ & 40.74 & 1.48 & $* *$ \\
\hline Cellulose\% & $14.05^{\mathrm{d}}$ & $29.55^{\mathrm{a}}$ & $23.37^{\mathrm{b}}$ & $30.44^{\mathrm{a}}$ & $24.21^{b}$ & $20.07^{c}$ & 21.47 & 2.32 & $* *$ \\
\hline Lignin\% & $9.70^{\mathrm{e}}$ & $16.81^{\mathrm{d}}$ & $15.02^{\mathrm{d}}$ & $22.24^{\mathrm{c}}$ & $25.14^{\mathrm{b}}$ & $33.86^{\mathrm{a}}$ & 19.34 & 1.41 & * \\
\hline ME MJ/kg/DM & $7.77^{\mathrm{b}}$ & $6.63^{c}$ & $8.81^{\mathrm{a}}$ & $6.60^{c}$ & $6.17^{\mathrm{c}}$ & $6.16^{c}$ & 6.88 & 0.14 & $*$ \\
\hline
\end{tabular}

a, b, c, and $d=$ Values on the same row with different superscripts differ significantly. SEM = Standard Error. LSD $=$ Least Significant Difference. NS = Not Significant. ${ }^{*}=$ Significant at $(\mathrm{P}<0.05) .{ }^{* *}=$ Highly Significant $(\mathrm{P}<0.001) . \mathrm{DM}=$ Dry Matter, OM $=$ Organic Matter, $\mathrm{CP}=\mathrm{Crude}$ Protein, $\mathrm{CF}=\mathrm{Crude}$ Fiber, $\mathrm{EE}=$ Ether Extract, NFE $=$ Nitrogen Free Extract, NDF $=$ Neutral Detergent Fiber, ADF $=$ Acid Detergent Fiber, ME $=$ Metabolizable Energy . 
Table 5. Comparison of nutritive value of the range herbage for the two grazing seasons.

\begin{tabular}{|c|c|c|c|c|c|c|c|c|c|c|c|c|c|}
\hline Season & DM\% & $\begin{array}{l}\text { Ash } \\
\%\end{array}$ & OM\% & СР\% & CF\% & EE\% & $\begin{array}{c}\text { NFEE } \\
\%\end{array}$ & $\begin{array}{c}\mathrm{NDF} \\
\%\end{array}$ & $\begin{array}{c}\text { Hemi } \\
\text { Cellulose } \\
\%\end{array}$ & $\begin{array}{c}\mathrm{ADF} \\
\%\end{array}$ & $\begin{array}{c}\text { Cellulose } \\
\%\end{array}$ & $\begin{array}{l}\text { Lignin } \\
\%\end{array}$ & $\begin{array}{c}\mathrm{ME} \\
\mathrm{MJ} / \mathrm{kg} / \mathrm{DM}\end{array}$ \\
\hline 2004 & $88.65^{b}$ & $10.94^{b}$ & $89.11^{\mathrm{a}}$ & $\begin{array}{l}\text { NS } \\
6.06\end{array}$ & $\begin{array}{c}\text { NS } \\
29.85\end{array}$ & $6.88^{\mathrm{a}}$ & $\begin{array}{c}\text { NS } \\
38.81\end{array}$ & $60.09^{\mathrm{a}}$ & $21.31^{\mathrm{a}}$ & $39.28^{b}$ & $23.73^{\mathrm{a}}$ & $15.45^{\mathrm{b}}$ & $12.45^{\mathrm{a}}$ \\
\hline 2005 & $89.77^{\mathrm{a}}$ & $11.73^{\mathrm{a}}$ & $88.42^{\mathrm{b}}$ & $\begin{array}{l}\text { NS } \\
6.21\end{array}$ & $\begin{array}{c}\text { NS } \\
29.88\end{array}$ & $4.96^{\mathrm{b}}$ & $\begin{array}{c}\text { NS } \\
39.60\end{array}$ & $54.67^{b}$ & $14.00^{\mathrm{b}}$ & $40.74^{\mathrm{a}}$ & $21.47^{b}$ & $19.24^{\mathrm{a}}$ & $11.44^{\mathrm{a}}$ \\
\hline $\begin{array}{l}\text { Overall } \\
\text { Mean }\end{array}$ & 89.21 & 11.34 & 88.77 & 6.13 & 29.87 & 5.92 & 39.20 & 57.39 & 17.66 & 40.00 & 22.60 & 17.35 & 11.95 \\
\hline SED & 1.580 & 1.12 & 0.90 & 0.56 & 1.42 & 0.56 & 1.63 & 1.38 & 2.81 & 1.75 & 2.58 & 1.50 & 0.69 \\
\hline Sign & * & ** & * & NS & NS & * & NS & * & $*$ & * & * & * & NS \\
\hline
\end{tabular}

$\mathrm{a}$ and $\mathrm{b}=$ Values on the same column with different superscripts differ significantly. SED $=$ Standard Error Differences. ${ }^{*}=$ Significant at $\mathrm{P}(<0.05){ }^{* *}=$ Significant at $(\mathrm{P}<0.01)$. NS $=$ Not Significant. DM = Dry Matter, OM = Organic Matter, $\mathrm{CP}=$ Crude Protein, $\mathrm{CF}=\mathrm{Crude}$ Fiber, EE $=$ Ether Extract, NFE $=$ Nitrogen Free Extract, NDF = Neutral Detergent Fiber, ADF = Acid Detergent Fiber, ME = Metabolizable Energy.

The variations in the nutritional value of these range herbage considerably differ within the season. From the first showers and rains, the grass sward is established and within the range of the first three weeks of age as from July throughout towards the end of the rainy or grazing season in October up to December (i.e. autumn and winter) varies. This seems to follow the natural or usual patterns under unimodel rainfall.

Most tropical and subtropical grassland have high nutritive value during the early growth, but their nutritive value declines rapidly with maturity [25]. Most shrubs and grasslands generally have high levels of crude protein and energy content throughout the grazing season [26]. In the present study, the experiment was conducted in the same scheduled time in the tow seasons. The grassland exhibited slight seasonal changes or variations in their nutritive value in each season and within the same season.

The dry matter (DM) apparently in the two seasons tends to increase towards the mid of September until it reached its maximum level in mid October, when all plant growth was achieved at maturity. Neutral detergent fiber (NDF), follow the same level trend of increase as dry matter. This finding goes in line with that of [18] and [26] who reported that as pasture advanced in growth, the dry matter, crude protein and lignin contents tend to increase with increasing stage of maturity. Towards the mid of November and mid of December, both dry matter and neutral detergent fiber increases ceased due to reduced growth in plant tissues.

The crude protein and lignin increase were significantly modified by the range grass age. The crude protein increase beings in early rains in July then starts to increase towards the mid September when it reached its highest level when range herbage was well established due to sufficient amount of rainfall and excellent growth of all range species. Apparently the lignin also follows the trend level of increase of crude protein with the advanced grass age but in less percentage. Moreover, the crude protein started to decline towards the mid of October, through November and December while lignin level tends to a continued increase as range species ages increase towards the mid November and mid December. Similar trends were observed by [16] that drastic reduction in crude protein in December (4.6\%) while the peak protein value was attained in September (8.2\%). [17] provided seasonal variations in grassland crude protein level (CP) as 60 - $80 \mathrm{Kg} \mathrm{CP} / \mathrm{ha}$ in wet season (September to October) while it may be reduced to 25 - $40 \mathrm{Kg} \mathrm{CP/ha} \mathrm{towards} \mathrm{December} \mathrm{and} \mathrm{end} \mathrm{of} \mathrm{January.}$

Apparently the metabolizable energy, (ME MJ/KgDM) was significantly higher in mid September (8.80 $\mathrm{MJ} / \mathrm{Kg}$ ) than in mid October $(6.76 \mathrm{MJ} / \mathrm{Kg})$ and mid December $(6.44 \mathrm{MJ} / \mathrm{Kg})$. Also Hemicellulose and Neutral detergent fiber (NDF) in the present study were comparatively higher in season 2004 than in 2005, which could be attributed to rainfall fluctuation. Nitrogen Free Ether Extracts and Ash findings were accordance with [16] revealing a total Ash of $16 \%$, and 39.9\% NFEE.

It can be stipulated that despite the variations in rainfall, as they gain maturity, most grasses were expected to lose their high nutritive value they had during their early stages of growth [26]. This implies that the range nutritive value tends to decrease towards the approach of the dry season (dry summer) and implies needs for measures for supplementary feeding to sustain livestock requirements because crude protein and metabolizable energy tends to be reduced as Lignin, hemi-cellulose and cellulose tend to increase with a drastic drop in crude protein levels towards the dry season. It is likely that the crude protein for much of the available herbage from Feb- 
ruary to (before rain) July would be low.

\section{Conclusion}

In conclusion, as herbage age increase towards the end of the grazing season (rainy season), its nutritive value drastically declined in terms of crude protein (CP) and metabolizable energy (MJ) content. The age affected nutritional status of grasslands. Crude protein (4.03\%) content decreased with the increased maturity stage of range grasses (27 weeks), accordingly the crude fiber (23.79\%) also was depressed by age increase. This in terms was ascribed to increase in lignin content (34.85\%). Also the nutritional status of the range site varies from season to season depending on the intensity of rainfall received. Therefore the animals have to graze in large areas before they can meet and satisfy their maintenance requirements.

\section{Acknowledgements}

This study was supported by University of Juba with the grant-in-aid from the former Directorate of Animal Resources Research Co-Operation, Sudan.

\section{References}

[1] Murdeh, A.W. and Gordon, J.S. (1992) Climate, in Tropical Pasture Research Principles and Methods. In: Show, N.N. and Breyen, W.W., Eds., Tropical Pasture Research Principles and Methods, Berkshire, England, 203-218.

[2] American Society of Range Management in Joins Committee with the Agricultural Board (1999) Basic Problem and Techniques in Range Research. Natal Academy of Science, Washington DC.

[3] Giriraj, A., Murthy, M.S.R. and Ramesh, B.R. (2008) Vegetation Composition, Structure and Patterns of Diversity: A Case Study from the Tropical Wet Evergreen Forests of the Western Ghats, India. Oxford University Press, New York.

[4] Fischer, G., Nachtergaele, F., Prieler, S., van Velthuizen, H.T., Verelst, L. and Wiberg, D. (2008) Global Agro-Ecological Zones Assessment Foragriculture (GAEZ 2008). Camb.

[5] Crowder, L.V. and Chedda, H.R. (1982) Herbage Quality and Nutritive Value. Tropical Grassland Husbandry, Longman Inc., New York, 349.

[6] Kamuanga, M.J., Somda, J., Sanon, Y. and Kagoné, H. (2008) Livestock and Regional Market in the Sahel and West Africa. Potentials and Challenges. SWAC-OECD/ECOWAS, Sahel.

[7] FAO (2011) World Livestock 2011—Livestock in Food Security. FAO, Rome.

[8] FAO (1996) World Livestock Production Systems: Current Status, Issues and Trends. In: Seré, C. and Steinfeld, H., Eds., FAO Animal Production and Health Paper 127, FAO, Rome, 217-238.

[9] Bertelsen, B.S., Faulkner, D.B., Buskirk, D.D. and Castree, J.W. (1993) Beef Cattle Performance and Forage Characteristics of Continuous, 6-Paddock, and 11-Paddock Grazing Systems. Journal of Animal Science, 71, 1381-1389.

[10] Mohamed Saleem, M.A. (1998) Nutrient Balance Patterns in African Livestock Systems. Agriculture, Ecosystems \& Environment, 71, 241-254. http://dx.doi.org/10.1016/S0167-8809(98)00144-3

[11] Minson, D.J. (1976) The Nutritive Value of Tropical Pastures. Journal of the Australian Institute of Agricultural Science, 37, 255-263.

[12] Keady, T.W.J., Marley, C.M. and Scollan, N.D. (2012) Grass and Alternative Forage Silages for Beef Cattle and Sheep: Effects on Animal Performance. Proceedings of the 16th International Silage Conference, Hämeenlinna, 2-4 July 2012, 210-228.

[13] Griff, D.B. (1974) The Agricultural System of the World: An Evolutionary Approach. Cambridge University Press, New York, 112-118.

[14] FAO (2011) Global Livestock Production Systems. Production Systems, Rome, 87-113.

[15] Wilson, R.T. and Clerk, S.E. (1995) Studies of the Livestock of Southern Darfur, Sudan. 1. The Ecology and Livestock Resources of the Area. Oxford University Press, New York, 185-198.

[16] Ahmed, F.A. and Ahmed, A.E. (1998) Plant Cover, Nutritive Value and Carrying Capacity of Rage Pasture in the Low Rainfall Woodland Savannah of Western Sudan. 43, 238-245.

[17] Hunting Technical Services Limited (1975) Savannah Development Project Phase II, Livestock and Range Investigations, Gahzala Gawazat. 21-23.

[18] Salih, F.A. (1987) The Effect of Maturity Stage on the Nutritive Value of Some Range Grasses in Low Rain Woodland Savannah Ranges. M.Sc. Thesis, University of Khartoum, Khartoum. 
[19] AOAC (1997) Association of Official Analytical Chemists International Official Methods of Analysis. 16th Edition, AOAC, Arlington.

[20] Shawkat, A.A. and Tautomu, E. (1999) Animal Nutrition: A Laboratory Manual. Laboratory of Animal Science, Shimane University, Shimane.

[21] Van Soest, P.J., Robertson, J.B. and Lewis, B.A. (1991) Methods for Dietary Neural Detergent Fiber and Non Starch Polysaccharides in Relation to Animal Nutrition. Journal of Diary Science, 74, 3583-3597. http://download.journals.elsevierhealth.com/pdfs/journals/0022-0302/PIIS0022030291785512.pdf

[22] MAFF, DAFS and DANI (1977) Ministry of Agriculture Food and Fisheries, Department of Agriculture Fisheries for Scotland, and Department of Agriculture for Northern Ireland, Technical Bulletin 33 H.M.S.O. London. MAFF, DAFS and DANI a Joint Bull.

[23] Voelkl Kristin, E. and Gerber Susan, B. (1998) Using SSPS for Windows, Data Analysis and Graphics. J. Dairy Sci., 74, 3583-3597.

[24] Duncan, D.B. (1955) Multiple Range and Multiple F-Tests. Biometrics, 11, 1-42. http://dx.doi.org/10.2307/3001478

[25] Khair, M., El Shantnaawi, J. and Mohawesh, Y.M. (2000) Seasonal Chemical Composition of Saltbush in Semi-Arid Grassland of Jordon. Journal of Range Management, 53, 211-214. http://dx.doi.org/10.2307/4003285

[26] Playne, M.J. (1980) Differences in Nutritional Value of Three Cuts of Buffel Grass for Sheep and Cattle. Proceedings of the Australian Society of Animal Production, 8, 511-516. 
Scientific Research Publishing (SCIRP) is one of the largest Open Access journal publishers. It is currently publishing more than 200 open access, online, peer-reviewed journals covering a wide range of academic disciplines. SCIRP serves the worldwide academic communities and contributes to the progress and application of science with its publication.

Other selected journals from SCIRP are listed as below. Submit your manuscript to us via either submit@scirp.org or Online Submission Portal.
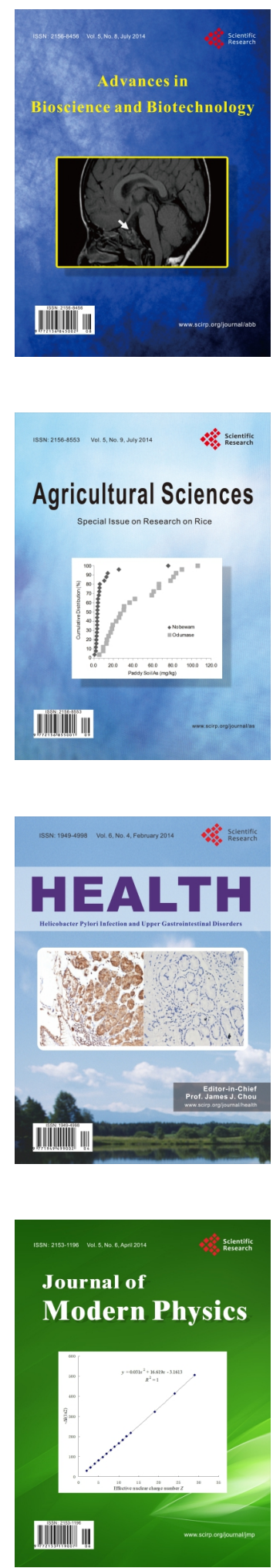
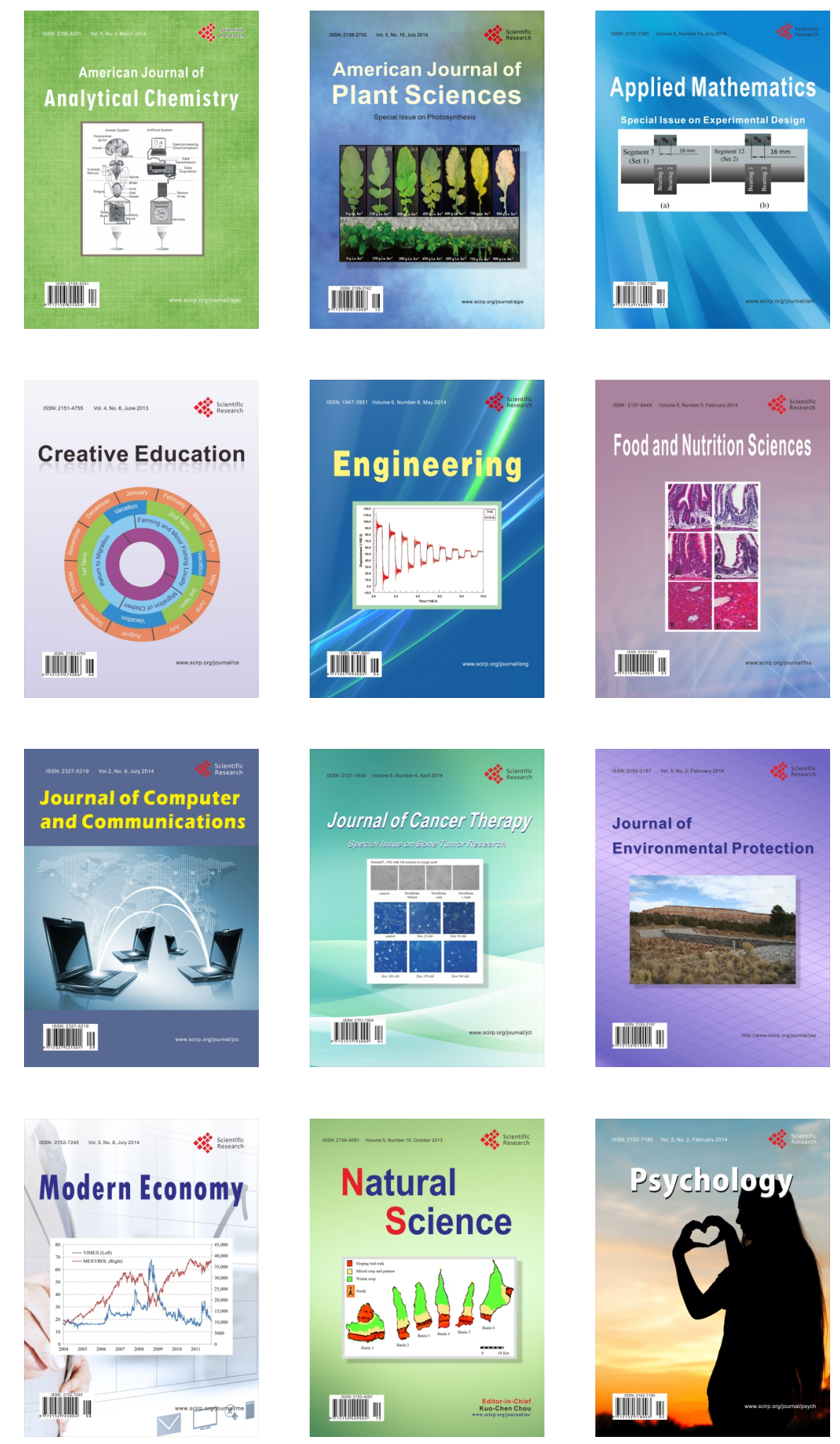